\title{
Neurally Adjusted Ventilatory Assist and Pressure Support Ventilation in Small Species and the Impact of Instrumental Dead Space
}

\author{
Francesca Campoccia Jalde ${ }^{a}$ Abdul Raoof Almadhoob ${ }^{b} \quad$ Jennifer Beck ${ }^{c-e}$ \\ Arthur S. Slutsky ${ }^{e, f}$ Michael S. Dunn ${ }^{c, d}$ Christer Sinderby ${ }^{e, f}$ \\ ${ }^{a}$ Department of Anaesthesiology and Intensive Care, Karolinska Hospital and Institute, Stockholm, Sweden; \\ ${ }^{b}$ University of Toronto Neonatal-Perinatal Integrated Fellowship Program, 'Women and Babies Program, \\ Sunnybrook Health Sciences Centre, ${ }^{\mathrm{d}}$ Department of Pediatrics, University of Toronto, ${ }^{e}$ Department of Critical \\ Care Medicine, Keenan Research Centre in the Li Ka Shing Knowledge Institute of St. Michael's Hospital, and \\ fDepartment of Medicine, University of Toronto, Toronto, Ont., Canada
}

\section{Key Words}

Neurally adjusted ventilatory assist $\cdot$ Pressure support ventilation $\cdot$ Diaphragm electrical activity $\cdot$ Instrumental dead space

\begin{abstract}
Background: Neurally adjusted ventilatory assist (NAVA) is a pneumatically-independent mode of mechanical ventilation controlled by diaphragm electrical activity (EAdi), and has not yet been implemented in very small species. Objectives: The aims of the study were to evaluate the feasibility of applying NAVA in very small species and to compare this to pressure support ventilation (PSV) in terms of ventilatory efficiency and breathing pattern, and evaluate the impact of instrumental dead space on breathing pattern during both modes. Methods: Nine healthy rats (mean weight $385 \pm$ $4 \mathrm{~g}$ ) were studied while breathing on PSV or NAVA, at baseline or with added dead space. Results: A clear difference in breathing pattern between NAVA and PSV was observed during both baseline and dead space, where PSV - despite similar EAdi and tidal volume as during NAVA - caused shortened inspiratory time $(p<0.05)$ and increased the respiratory rate $(p<0.05)$. A higher minute ventilation $(p<0.05)$ in
\end{abstract}

order to reach the same arterial $\mathrm{CO}_{2}$ was observed. Ineffective inspiratory efforts occurred only during PSV and decreased with the dead space. Conclusion: This study demonstrates, in a small group of animals, that NAVA can deliver assist in very small species with a higher efficiency than PSV in terms of eliminating $\mathrm{CO}_{2}$ for a given minute ventilation.

Copyright $\odot 2009$ S. Karger AG, Basel

\section{Introduction}

Patient-ventilator asynchrony has been demonstrated in infants and in relatively small animals breathing on conventional, pneumatically-controlled mechanical ventilation [1-3]. Neurally adjusted ventilatory assist (NAVA) is a new mode of mechanical ventilation where the assist delivery is controlled by diaphragm electrical activity (EAdi) [4], which is a pneumatically-independent controller signal. To date, NAVA has been implemented successfully in White New Zealand rabbits with a weight of approximately $3 \mathrm{~kg}[2,5,6]$, and has been shown to provide a more efficient and synchronous ventilation. No studies have yet been performed with the application of NAVA in very small species.

\section{KARGER}

() 2009 S. Karger AG, Base

Fax +41613061234 E-Mail karger@karger.ch www.karger.com www.karger.com/neo
Jennifer Beck, PhD

Keenan Research Centre in the Li Ka Shing Knowledge Institute, St. Michael's Hospital 30 Bond Street, Queen Wing 4-072

Toronto, ON M5B 1W8 (Canada)

Tel. +1 416880 3664, Fax +1 416760 7518, E-Mail beckj@smh.toronto.on.ca 
Today, pressure support ventilation (PSV) is one of the modes of providing partial ventilatory assist during spontaneous breathing. Different from NAVA, PSV is triggered and cycled off by pneumatic sensors responding to airway pressure $\left(\mathrm{P}_{\mathrm{aw}}\right)$, flow, and/or volume, and delivers a constant targeted level of pressure. To our knowledge, very few physiological trials of PSV in very small species have been performed [7].

Ironically, pressure- or flow-sensing devices are often inserted into the respiratory circuit between the endotracheal tube and y-piece in order to improve monitoring of tidal volume (Vt) and/or to better synchronize the assist delivery during PSV in neonates. These devices typically add a dead space volume of about $0.8-1.0 \mathrm{ml}$, and although breathing pattern responses have been described, no papers to our knowledge have described the impact of adding a flow-sensing device on neural respiratory drive.

The aim of the present study was to evaluate the feasibility of applying NAVA in tracheostomized (i.e., absence of leaks) very small species (Sprague-Dawley rats) weighing less than $400 \mathrm{~g}$ and to compare this to ventilatory efficiency and breathing pattern with PSV, while breathing with and without an added $(0.8 \mathrm{ml})$ dead space.

\section{Methods}

The Animal Care and Use Committee of St. Michael's Hospital, Toronto, Ont., Canada, approved the protocol.

\section{Animal Preparation and Instrumentation}

Nine adult male Sprague-Dawley rats (Charles River Labs, St. Constant, Que., Canada) with a mean body weight of $385 \pm$ $4 \mathrm{~g}$, were studied. The animals were initially anesthetized with isoflurane in $\mathrm{O}_{2}(100 \%)$ and titrated until the animals were motionless and unresponsive to stimulus. The rats were then injected with intraperitoneal ketamine $(100 \mathrm{mg} / \mathrm{kg})$ and xylazine $(10$ $\mathrm{mg} / \mathrm{kg}$ ). Following premedication, anesthesia was maintained throughout the protocol via continuous intravenous infusion of ketamine $(25 \mathrm{mg} / \mathrm{kg} / \mathrm{h})$, xylazine $(2.5 \mathrm{mg} / \mathrm{kg} / \mathrm{h})$ via the tail vein. With this anesthetic protocol, the animals maintained spontaneous ventilation.

A tracheostomy was performed using a 14-gauge tube and secured in place with silk suture. The femoral artery was cannulated to obtain blood for arterial blood gases (Ciba-Corning Model 248; Bayer, Leverkusen, Germany). Transcutaneous pulse oximetry was used to monitor heart rate and arterial oxygen saturation (NONIN 8600 VTM; Nonin Medical Inc., Plymouth, Minn., USA) at the paw. Body temperature was measured with a rectal probe and maintained at $37 \pm 0.5^{\circ} \mathrm{C}$ using a heating pad. Flow, $\mathrm{Vt}$ and $\mathrm{P}_{\mathrm{aw}}$ were measured through a pneumotachograph (Hans Rudolph 8411 series, linear between 0-10 1/min, $1.3 \mathrm{ml}$ dead space) placed between the tracheostomy and the $y$-piece of the respiratory circuit throughout the entire study. Since the anatomical dead space of the upper airway structures (approx. $0.5 \mathrm{ml}$ ) was bypassed by the tracheotomy, the effective net dead space of the pneumotach was around $0.8 \mathrm{ml}$. Volume was obtained by integration of the flow signal.

EAdi was recorded from an array of miniaturized electrodes, spaced $4 \mathrm{~mm}$ apart and mounted on a 5.6-french esophageal catheter. This interelectrode distance fulfilled criteria necessary for the double subtraction method $[8,9]$ to process EAdi.

The accurate positioning of the EAdi electrodes was confirmed by an online display of ECG and correlograms of EAdi signals along the electrode array [8]. A detailed description of electrode positioning is provided in Beck et al. [2]. Standardized and automated signal processing algorithms were implemented on the EAdi signal, as previously described [8-10].

Method for PSV and NAVA

NAVA and PSV were both applied with a modified Servo 300 ventilator (Maquet Critical Care, Solna, Sweden).

PSV was used with the standard features of the ventilator. The flow trigger in PSV (neonatal setting) was set to a medium sensitive flow trigger (around $0.35 \mathrm{l} / \mathrm{min}$ ), which in this animal model, does not cause auto-triggering, and the default cycling-off was at $5 \%$ of peak inspiratory flow (nonadjustable).

During NAVA, the pressure delivery was controlled by the EAdi waveform, processed according to standardized algorithms [9]. While the ventilator was set in 'pressure support/CPAP mode', the EAdi overrode the built-in pressure support algorithms. During NAVA, the EAdi signal was used to trigger and cycle-off the assist. EAdi was also the controller signal for the amount of pressure delivered during inspiration and was multiplied by a proportionality factor known as the NAVA level to adjust the level of assist [4]. The NAVA trigger was set to a level where it was not triggering on random noise signals (this fixed trigger level was determined during paralysis in a separate group of animals). The NAVA off-cycling was set to $80 \%$ of peak EAdi. Trigger and cycling-off variables were not altered throughout the study.

In both modes, the rise time was set to $5 \%$, was similar for all animals and was not adjusted during the experiment. Hence, except for the 'external' processing of the NAVA signal, all digital, electronic and pneumatic interventions in the Servo 300 were identical for both NAVA and PSV.

Bench testing of Servo 300 has demonstrated clearly acceptable performance for the infant mode [11]. No such evaluation has been published in the neonatal mode.

\section{Experimental Protocol}

Following instrumentation, animals were first ventilated with NAVA (at a fixed NAVA level of $0.8 \mathrm{~cm} \mathrm{H}_{2} \mathrm{O}$ /arbitrary units of EAdi). This was necessary because we wanted to match the peak pressures in both NAVA and PSV. Since NAVA is not pressuretargeted and delivers pressure in proportion to EAdi, and considering that each individual rat has a different EAdi and response to dead space, the peak pressure during NAVA was unpredictable. Since PSV allows specific targeting of peak pressure, peak pressures could be matched in the 2 nd period with PSV. In both modes, a dead space was applied in random order and placed between the endotracheal tube and y-piece.

NAVA and PSV levels were not altered between periods with and without dead space. $\mathrm{FiO}_{2}$ was 0.5 and PEEP was $1.5[1.5 ; 2.0$ 
Table 1. Neural breathing pattern and arterial $\mathrm{CO}_{2}$ for the different ventilatory conditions

\begin{tabular}{|c|c|c|c|c|c|}
\hline Variable & NAVAbl & NAVAds & PSVbl & PSVds & $\begin{array}{l}\text { ANOVA } \\
\text { p value }\end{array}$ \\
\hline $\mathrm{PaCO}_{2}, \mathrm{~mm} \mathrm{Hg}$ & $48.5(44 ; 54)$ & $55.5(53.5 ; 65)^{\mathrm{c}}$ & $51(46 ; 55)$ & $59.5(59 ; 61.5)^{\mathrm{d}}$ & 0.002 \\
\hline IXEAdi, AU & $8.5(7.4 ; 12.3)$ & $11.8(8.9 ; 14.6)^{\mathrm{c}}$ & $10.4(7.3 ; 13.6)$ & $12.9(9.0 ; 17.3)^{\mathrm{d}}$ & 0.015 \\
\hline $\mathrm{Ti}, \mathrm{ms}$ & $474(450 ; 512)$ & $428(391 ; 486)^{\mathrm{c}}$ & $340(306 ; 382)^{\mathrm{a}}$ & $266(235 ; 294)^{\mathrm{b}, \mathrm{d}}$ & $<0.001$ \\
\hline Ti/Ttot & $0.53(0.47 ; 0.60)$ & $0.52(0.49 ; 0.61)^{\mathrm{C}}$ & $0.41(0.38 ; 0.49)^{\mathrm{a}}$ & $0.41(0.34 ; 0.44)^{\mathrm{b}}$ & 0.002 \\
\hline $\mathrm{Vt} / \mathrm{Ti}, \mathrm{l} / \mathrm{sec}$ & $5.3(4.4 ; 6.2)$ & $6.4(5.9 ; 6.8)^{\mathrm{c}}$ & $7.7(6.7 ; 8.8)^{\mathrm{a}}$ & $9.6(9.4 ; 11.2)^{\mathrm{b}, \mathrm{d}}$ & $<0.001$ \\
\hline
\end{tabular}

Values are reported as medians (25-75\%). bl = Baseline condition; ds = dead space condition; $\mathrm{PaCO}_{2}=$ partial pressure of carbon dioxide in the arterial blood; IXEAdi = mean inspiratory diaphragm electrical activity; AU = arbitrary units.

${ }^{a}$ Post hoc comparison of NAVAbl vs. PSVbl, $\mathrm{p}<0.05$. ${ }^{\mathrm{b}}$ Post hoc comparison of NAVAds vs. PSVds, $\mathrm{p}<0.05$. ${ }^{\mathrm{c}}$ Post hoc comparison of NAVAbl vs. NAVAds, $\mathrm{p}<0.05 .{ }^{\mathrm{d}}$ Post hoc comparison of PSVbl vs. PSVds, $\mathrm{p}<0.05$.

(median with 25 and $75 \%$ quartiles)] $\mathrm{cm} \mathrm{H}_{2} \mathrm{O}$ and not changed throughout the protocol.

Each period of NAVA and PSV, with and without dead space, lasted $10 \mathrm{~min}$, which was substantially longer than the response time for chemoreceptor stimulus [12]. The last $5 \mathrm{~min}$ were recorded. Arterial blood was drawn and analyzed at the end of each period.

\section{Off-Line Analysis}

The last minute of each period was analyzed to quantify flow, $\mathrm{Vt}, \mathrm{P}_{\mathrm{aw}}$, neural respiratory rate (nRR), ventilator respiratory rate and mean inspiratory EAdi on a breath-by-breath basis. Minute ventilation (VE) was calculated as the product of the Vt and ventilator respiratory rate for the assisted breaths.

\section{Statistics}

Because some of the measured variables were not normally distributed, results are reported as medians with the 25 and $75 \%$ quartiles. For the 4 conditions, multiple comparisons were performed with repeated measures ANOVA on ranks using the Student-Newman-Keuls method for pair-wise multiple comparisons. Relationships were determined with the Pearson product-moment correlation. $\mathrm{p}<0.05$ was considered significant.

\section{Results}

All runs were successfully completed in all animals.

\section{Baseline (No Dead Space but on Mechanical}

Ventilation with the Pneumotach Placed in Line)

$\mathrm{PaCO}_{2}$ was slightly higher than normal during the baseline periods with both NAVA and PSV; however, no difference was observed between the modes (table 1). $\mathrm{PaO}_{2}$ during the baseline period for PSV and NAVA was
$183.0(144.5 ; 237.5)$ and $189.0(167.5 ; 207.5) \mathrm{mm} \mathrm{Hg}$, respectively, and not statistically different. There was no difference in mean inspiratory EAdi between the NAVA and PSV modes (table 1).

The mean $\mathrm{P}_{\mathrm{aw}}$ during the baseline periods of NAVA and PSV was $3.5(3.0 ; 4.6)$ and $3.4(2.8 ; 4.1) \mathrm{cm} \mathrm{H}_{2} \mathrm{O}$, respectively. The corresponding peak values were 5.5 (5.2; 8.4) and $7.3(5.9 ; 9.0) \mathrm{cm} \mathrm{H}_{2} \mathrm{O}$. There were no statistical differences for mean or peak $\mathrm{P}_{\mathrm{aw}}$ between the NAVA and PSV periods.

No difference was observed between NAVA and PSV with regards to $\mathrm{Vt}$, whereas significant differences were observed (repeated measures ANOVA on ranks) for inspiratory time $(\mathrm{Ti} ; \mathrm{p}<0.001)$, duty cycle $[\mathrm{Ti} / \mathrm{Ttot}$ (total breath duration); $\mathrm{p}<0.001$, mean inspiratory flow (Vt/ Ti; $\mathrm{p}=0.001)$, nRR $(\mathrm{p}<0.001)$ and VE $(\mathrm{p}=0.002)$. Post hoc comparison showed significantly lower $\mathrm{Ti}(\mathrm{p}<0.05)$ and $\mathrm{Ti} / \mathrm{Tt}(\mathrm{p}<0.05)$, and higher $\mathrm{Vt} / \mathrm{Ti}(\mathrm{p}<0.05)$ and $\mathrm{nRR}$ $(\mathrm{p}<0.05)$, during PSV in comparison to NAVA. The results for Vt, nRR and VE are depicted in figure 1 and the results for $\mathrm{Ti}, \mathrm{Ti} / \mathrm{Tt}$, and $\mathrm{Vt} / \mathrm{Ti}$ are presented in table 1 .

During baseline PSV, ineffective efforts were observed (defined as the presence of EAdi that did not trigger the ventilator). The number of ineffective efforts ranged between 1 and 5 per min (median 2.5 per min) and were observed in 4 of the 9 rats. No ineffective efforts were observed during NAVA.

Despite the ineffective inspiratory efforts during PSV at baseline, VE was $26 \%(6 ; 36)$ higher $(\mathrm{p}<0.05)$ during PSV than during NAVA (fig. 1). 


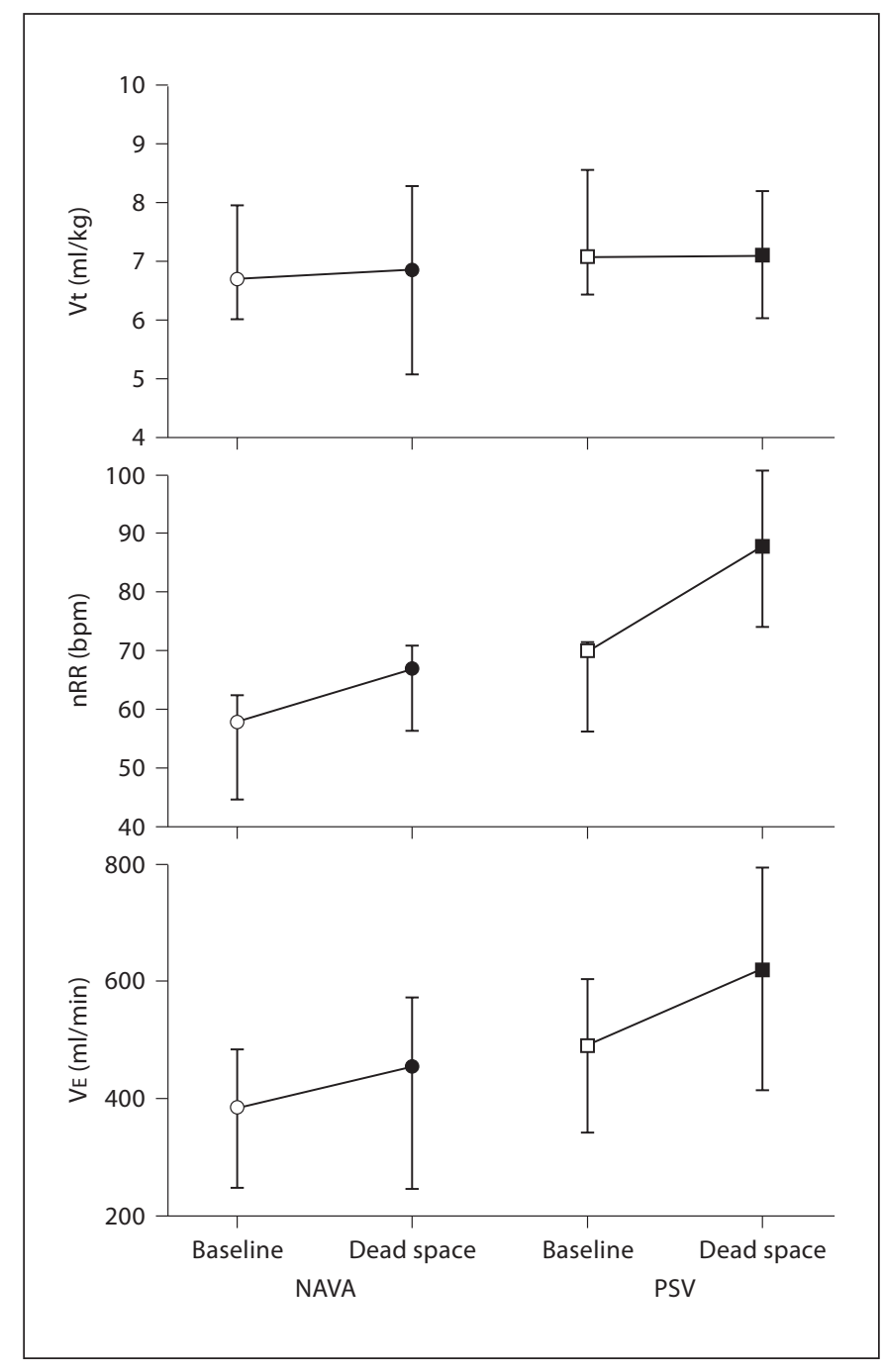

Fig. 1. Vt, $n R R$, and minute ventilation (VE) during NAVA baseline periods (circles), PSV (squares) baseline periods (empty symbols) and during periods with dead space (solid symbols). Data are presented as medians with 25 and $75 \%$ quartiles.

Dead Space (on Mechanical Ventilation with the Pneumotach and $0.8 \mathrm{ml}$ Dead Space Placed in Line)

With the addition of dead space, $\mathrm{PaCO}_{2}$ increased $(\mathrm{p}<0.001)$ to similar levels during both NAVA $(\mathrm{p}<0.05)$ and PSV $(\mathrm{p}<0.05)$ as presented in table $1 . \mathrm{PaO}_{2}$ values during NAVA and PSV with added dead space were 192.5 $(144.0 ; 258.5)$ and $208.5(187.0 ; 243.5) \mathrm{mm} \mathrm{Hg}$, respectively, and not different from baseline values.

Adding the dead space increased mean inspiratory EAdi $(\mathrm{p}=0.015)$ during NAVA $(\mathrm{p}<0.05)$ and PSV $(\mathrm{p}<$ 0.05 ) to similar levels (table 1). During NAVA, the dead space-induced changes in EAdi were correlated to chang- es in $\mathrm{P}_{\mathrm{aw}}(\mathrm{r}=0.71, \mathrm{p}=0.030)$ producing an increase in $\mathrm{P}_{\mathrm{aw}}$ (above PEEP) by $8 \%(4-17 \%, p=0.008)$. No correlation between EAdi and $\mathrm{P}_{\mathrm{aw}}$ or increase in $\mathrm{P}_{\mathrm{aw}}$ was observed when dead space was added during PSV.

Vt was not different between NAVA and PSV and not changed compared to baseline after the addition of dead space (fig. 1). The addition of dead space increased $n R R$ by 9 breaths per minute $(\mathrm{bpm})$ during NAVA $(\mathrm{p}<0.05)$ and $18 \mathrm{bpm}$ during PSV $(\mathrm{p}<0.05)$. Similar to baseline conditions, nRR remained significantly higher during PSV compared to NAVA ( $p<0.05$; fig. 1$)$. The addition of dead space increased VE during both NAVA $(\mathrm{p}<0.05)$ and PSV $(\mathrm{p}<0.05)$. VE was still 25\% $(10 ; 48)$ higher $(\mathrm{p}<$ 0.05 ) during PSV compared to NAVA (fig. 1).

The application of the dead space shortened Ti during both NAVA $(\mathrm{p}<0.05)$ and PSV $(\mathrm{p}<0.05)$. Ti during PSV was still shorter than during NAVA $(\mathrm{p}<0.05$; table 1$)$. $\mathrm{Ti} / \mathrm{Tt}$ was minutely decreased during NAVA $(\mathrm{p}<0.05)$ and was not affected by the dead space during PSV. Ti/Tt was still significantly lower during PSV compared to NAVA $(\mathrm{p}<0.05)$ as presented in table 1 . Dead space increased Vt/Ti significantly, both during NAVA $(\mathrm{p}<0.05)$ and PSV $(\mathrm{p}<0.05)$. With dead space, Vt/Ti was still higher during PSV than during NAVA $(\mathrm{p}<0.05$; table 1$)$.

In PSV mode, only 1 rat demonstrated ineffective inspiratory efforts ( $3 / \mathrm{min})$ after the addition of dead space. No ineffective inspiratory efforts occurred during NAVA.

\section{Discussion}

The present study is the first to demonstrate the feasibility of NAVA in a very small species of $<400$ g body weight. With the ventilator and settings used, the results show a clear difference in breathing pattern between NAVA and PSV, where PSV - despite similar EAdi and Vt as NAVA - shortens $\mathrm{Ti}$, increases $\mathrm{nRR}$ and requires higher VE in order to reach the same $\mathrm{PaCO}_{2}$ level. Asynchrony was observed only during PSV, and was actually reduced when a dead space - equal in size to a flow-sensing device - was added to the respiratory circuit.

Despite the fact that the $\mathrm{PaCO}_{2}$ levels between NAVA and PSV were not different, VE was about $25 \%$ higher during PSV than during NAVA, both without and with dead space. Similar $\mathrm{PaCO}_{2}$, but higher VE, during PSV suggests that the efficiency in eliminating $\mathrm{PaCO}_{2}$ was lower compared to NAVA. One important factor affecting the $\mathrm{CO}_{2}$ elimination efficiency is the relationship between $\mathrm{Vt}$ and dead space. In the present study, all animals 
were their own control and were ventilated with the same respiratory circuit, only changing digital controllers to control the assist. The added dead space was the same during both NAVA and PSV. Hence, there were no differences in dead space volumes that could explain the worsened $\mathrm{CO}_{2}$ elimination efficiency during PSV. There were no differences in Vt between NAVA and PSV during breathing, with or without dead space. Hence, the effect of $\mathrm{Vt}$ to dead space ratio does not explain the worsened $\mathrm{CO}_{2}$ elimination efficiency during PSV, when compared to NAVA.

With regard to the measured Vt and respiratory rates in the present study, previous studies in healthy awake unrestrained rats breathing room air report Vt of about 4 and $7 \mathrm{ml} / \mathrm{kg}$ and breathing frequencies around $90 \mathrm{bpm}$ [7, 13-19]. Lai and Hildebrandt [20] described that sedatives lowered breathing frequency, whereas no influence on Vt was found $(7.3 \mathrm{ml} / \mathrm{kg})$. Hence, in the present study, the baseline Vt of about 6.7 (NAVA) and 7.1 (PSV) $\mathrm{ml} / \mathrm{kg}$ was within the previously reported normal range. Respiratory rates of 58 (NAVA) and 67 (PSV) bpm were within the range that has previously been described in PSV at a similar level of assist [7]. It is therefore likely, similar to the study of Lai and Hildebrandt [20], that the influence of sedatives on the respiratory rate and VE could explain, in part, the elevated $\mathrm{PaCO}_{2}$ at baseline in both modes of ventilation.

Another possible explanation for the lowered $\mathrm{CO}_{2}$ elimination efficiency during PSV, compared to NAVA, could be related to different Ti. Knelson et al. [21] demonstrated in dogs that increased length of the end inspiratory pause was followed by an increase in alveolar ventilation and a reduction in $\mathrm{PaCO}_{2}$. Mercat et al. [22] showed that extending $\mathrm{Ti}$ enhances $\mathrm{CO}_{2}$ elimination in human patients with acute respiratory distress syndrome. Recent work by Aström et al. [23] also showed that decreasing the mean distribution time during mechanical ventilation - either by decreasing the Ti time or by decreasing the pause time - worsened $\mathrm{CO}_{2}$ elimination. The shorter Ti during PSV may, therefore, be one explanation for the worsened efficiency of $\mathrm{CO}_{2}$ elimination in comparison to NAVA.

In the present study, nRR was clearly higher during PSV than during NAVA, regardless of whether dead space was added or not. Moreover, the increase in $\mathrm{nRR}$ due to added dead space during PSV was twice that observed during NAVA, even though increases in $\mathrm{PaCO}_{2}$ were similar. This could be explained by the differences in assist delivery and off-cycling algorithms between PSV and NAVA. Studies have shown that increased inspiratory flow rates delivered by the ventilator are associated with increasing breathing frequency [24]. During PSV, the inspiratory flow $(\mathrm{Vt} / \mathrm{Ti}$ ) was clearly higher (due to a shorter Ti) than during NAVA. Also, similar EAdi during NAVA and PSV suggests that the difference in flow was due to differences in how the assist was delivered and not due to differences in inspiratory effort. PSV is designed to reach the target pressure level as early as possible by maximizing the delivery of inspiratory flow at inspiratory onset, whereas NAVA is designed to deliver a pressure in proportion to effort which usually results in a saw-tooth shaped pressure profile where flow rates are more modest at onset of inspiratory effort.

Besides the initial flow rate, the duration of the assisted breath - in relation to the neural breathing cycle - has been shown to have an impact on respiratory rate [25], where a prolonged duration of assist into neural exhalation will act to slow the nRR [1], and a too short delivered breath will decrease neural exhalation time and, hence, promote an increase in respiratory rate [25]. In the present study, the assist period was longer during NAVA than during PSV, as evidenced by longer Ti and higher Ti/Tt during NAVA. NAVA was set to cycle off at $80 \%$ of peak EAdi, which, although this is beyond peak inspiratory effort, did not delay off-cycling to occlude expiratory flow at end-inspiration.

With regards to neonatal mechanical ventilation, only one study has addressed changing cycling-off criteria in very premature infants. This study demonstrated that expiratory asynchrony, expressed as triggered inflation extending into expiration, was reduced from 96 to $10 \%$ of the total number of triggered breaths when cycling-off criteria were adjusted from 0 to $25 \%$ of peak inspiratory flow, respectively [26]. Since this adjustment is arbitrary, mechanical inflation may persist into expiration, or on the other hand, may cease too early, as recently demonstrated [3] in neonates. In the present study, the default cycling-off criteria of 5\% also appeared to be too early compared to neural off-cycling. Appropriate cycling-off during PSV is, hence, an unresolved issue; therefore, more studies on patient-ventilator synchrony are required.

With regards to the physiological response to added dead space, a puzzling finding of the present study was that the application of the dead space only increased $n R R$, but not Vt. Previous works in healthy adult unrestrained conscious [15-17] and anesthetized rats [15, 27], not mechanically ventilated, show that $\mathrm{CO}_{2}$-induced increases in VE typically result from increases in both $\mathrm{Vt}$ and breathing frequency. Although we observed an expected 
increase in VE and respiratory drive with the added dead space in our study, the initial increase in dead space due to the pneumotach itself at baseline (see Methods) could have shifted the relative response of VT and $\mathrm{nRR}$, which may explain the lack of $\mathrm{Vt}$ response to the increased $\mathrm{CO}_{2}$ in the 2nd part of the study. Anesthesia has been found to blunt the ventilatory response to $\mathrm{CO}_{2}[15,27-29]$. The age of the rat has also been shown to affect the ventilatory responses to $\mathrm{CO}_{2}$ [29], as well as a tracheostomy [15]. Lai et al. [15] demonstrated that sedation in tracheostomized rats reduced respiratory rates, whereas little effect was observed on Vt. However, they showed that, although the ventilatory response to $\mathrm{CO}_{2}$ in unrestrained spontaneously breathing rats with tracheostomy was reduced, $\mathrm{VE}$ increased due to increases in both $\mathrm{Vt}$ and respiratory rates. A plausible explanation for the increase in VE as a result of increases in respiratory rates with maintained $\mathrm{Vt}$ in the present study could have been the implementation of mechanical ventilation. No studies of adding dead space during synchronized mechanical ventilation have been performed in either small rodents or extremely low birth weight neonates.

In the present study, almost half of the animals showed ineffective inspiratory efforts during PSV, whereas no EAdi breaths were missed during NAVA. Although one could claim that the incidence of ineffective inspiratory efforts during PSV was relatively low and that it indicates a good performance of the conventional trigger and cycling, one must consider that these animals were healthy and tracheotomized, which are actually ideal conditions for pneumatic triggering. During PSV, insertion of the dead space reduced the amount of ineffective inspiratory efforts despite the large increase in breathing frequency. Normally, one would expect an increase in breathing frequency to challenge the trigger and cycling-off capability of the ventilator and increase the incidence of ineffective inspiratory efforts during PSV. However, as demonstrated in the present study, the addition of dead space increased respiratory drive; therefore, an increased inspiratory effort for the same trigger threshold was likely the factor responsible for the reduced ineffective efforts.

\section{Study Limitations}

In the present study, we opted for fixed trigger settings in both modes (EAdi trigger for NAVA and flow trigger for PSV). Both were set at levels that did not induce autotriggering. Admittedly, either the EAdi and/or the flow triggers could have been set more sensitively (or another ventilator could have been used); however, this would have jeopardized the standardization of the protocol. Additionally, except for the external processing of the EAdi signal, all electronic and mechanical delays were identical for the 4 conditions since the same ventilator and circuitry were used. Nonetheless, the findings of the present study could be specific to the ventilator rather than the mode.

Another limitation of the present study is that the cycling-off criteria during PSV was bound to a fixed (by default) cycling-off criteria of $5 \%$ of peak inspiratory flow and was not adjustable. This could affect the likelihood of obtaining synchrony and the length of ventilator Ti. In a recent publication where the EAdi waveform was compared to the $\mathrm{P}_{\mathrm{aw}}$ waveform in premature infants on PSV, cycling-off at $15 \%$ of peak inspiratory flow was too early, as judged by the termination of the assisted breath prior to the peak of EAdi being reached [3].

The present study could be criticized for randomizing the application of dead space rather than randomizing the mode. Ventilator mode affects breathing pattern (i.e., how or how much assist is delivered), and this occurs rapidly $[1,30]$ and is assumed to be strongly influenced by reflexes [25, 30-32]. Consequently, the order in which modes are applied should not affect the differences in breathing pattern that were observed.

Regarding the relatively small sample size used in the present study, previous studies on breathing patterns in rats and acute $\mathrm{CO}_{2}$ challenge [7] typically have used 6-8 animals. This number of animals was sufficient to detect differences related to $\mathrm{CO}_{2}$ challenge. Based on this, we decided to study 9 animals, anticipating that this would be sufficient in a cross-over study design.

\section{Conclusion}

This study, in a small group of animals, demonstrates that NAVA can deliver ventilatory assist in very small species with a higher efficiency in terms of eliminating $\mathrm{CO}_{2}$ for a given minute ventilation than during PSV, with the ventilator settings used in the current study. The study also shows that during PSV, a dead space of $0.8 \mathrm{ml}$ reduces ineffective inspiratory efforts, but at the expense of increased respiratory drive. It should be noted that the results of the present study pertain to a small animal model with size being the challenge. Although clear and consistent differences were observed between PSV and NAVA, these results may not necessarily pertain to human premature infants with immature respiratory control. 


\section{Acknowledgements}

We are indebted to Mr. Norman Comtois for technical assistance. The study was supported by the R. Samuel McLaughlin Foundation and The Keenan Foundation. J.B. was supported by the NIH No. 1 R21 HD45047-01.

\section{Disclosure}

Drs. Beck and Sinderby have made inventions related to neural control of mechanical ventilation that are patented. The licenses for these patents belong to Maquet Critical Care. Future commercial uses of this technology may provide financial benefit to Drs. Beck and Sinderby through royalties. Drs. Beck and Sinderby each own $50 \%$ of Neurovent Research Inc. (NVR). NVR is a research and development company that builds equipment and catheters for research studies. NVR has a consulting agreement with Maquet Critical Care. Dr. Slutsky is a consultant to Maquet Medical and is compensated for this work.

\section{References}

1 Beck J, Tucci M, Emeriaud G, Lacroix J, Sinderby C: Prolonged neural expiratory time induced by mechanical ventilation in infants. Pediatr Res 2004;55:747-754.

2 Beck J, Campoccia F, Allo JC, Brander L, Brunet F, Slutsky AS, Sinderby C: Improved synchrony and respiratory unloading by neurally adjusted ventilatory assist (NAVA) in lung-injured rabbits. Pediatr Res 2007;61: 289-294.

3 Beck J, Reilly MC, Grasselli G, Mirabella L, Slutsky AS, Dunn MS, Sinderby C: Patientventilator interaction during neurally adjusted ventilatory assist in very low birth weight infants. Ped Res 2009;65:663-668.

$\checkmark 4$ Sinderby C, Navalesi P, Beck J, Skrobik Y, Comtois N, Friberg S, Gottfried SB, Lindström L: Neural control of mechanical ventilation in respiratory failure. Nat Med 1999; 5:1433-1436

5 Allo JC, BeckJC, Brander L, Brunet F, Slutsky AS, Sinderby CA: Influence of neurally adjusted ventilatory assist and positive end-expiratory pressure on breathing pattern in rabbits with acute lung injury. Crit Care Med 2006;34:2997-3004.

6 Beck J, Brander L, Slutsky AS, Reilly MC, Dunn MS, Sinderby C: Non-invasive neurally adjusted ventilatory assist in rabbits with acute lung injury. Intensive Care Med 2008; 34:316-323.

$\checkmark 7$ Goodyear-Bruch C, Long LR, Simon P, Clancy RL, Pierce JD: Pressure-support ventilation and diaphragm shortening in the rat model. AANA J 2005;73:277-283.

-8 Beck J, Sinderby C, Lindström L, Grassino A: Influence of bipolar esophageal electrode positioning on measurements of human crural diaphragm electromyogram. J Appl Physiol 1996;81:1434-1449.

-9 Sinderby C, Beck J, Lindström L, Grassino A: Enhancement of signal quality in esophageal recordings of diaphragm EMG. J Appl Physiol 1997;82:1370-1377.

- 10 Aldrich T, Sinderby C, McKenzie D, Estenne M, Gandevia S: ATS/ERS statement on respiratory muscle testing - electrophysiologic techniques for the assessment of respiratory muscle function. Am J Respir Crit Care Med 2002;166:518-624.
11 Nishimura M, Hess D, Kacmarek RM: The response of flow-triggered infant ventilators. Am J Respir Crit Care Med 1995;152:19011909.

12 Smith CA, Rodman JR, Chenuel BJ, Henderson KS, Dempsey JA: Response time and sensitivity of the ventilatory response to $\mathrm{CO}_{2}$ in unanesthetized intact dogs: central vs. peripheral chemoreceptors. J Appl Physiol 2006;100:13-19.

13 Palacek F: Measurement of ventilatory mechanics in the rat. J Appl Physiol 1969;27: 149-156.

14 Pappenheimer JR: Sleep and respiration of rats during hypoxia. J Physiol 1977;266:191207.

15 Lai YL, Tsuya Y, Hildebrandt J: Ventilatory responses to acute $\mathrm{CO}_{2}$ exposure in the rat. J Appl Physiol 1978;45:611-618.

16 Walker BR, Adams EM, Voelkel NF: Ventilatory responses of hamsters and rats to hypoxia and hypercapnia. J Appl Physiol 1985 59:1955-1960.

17 Walker JK, Jennings DB: Ventilatory and metabolic effects of hypercapnia in conscious rats: AVP V1 receptor block. Can J Physiol Pharmacol 1998;76:361-366.

18 Poole TB (ed): The UFAW Handbook on the Care and Management of Laboratory Animals, ed 6. Harlow, Longman Scientific \& Technical, 1986.

19 Harkness JE, Wagner JE: The Biology and Medicine of Rabbits and Rodents, ed 3. Philadelphia, Lea and Febiger, 1989.

20 Lai YL, Hildebrandt J: Respiratory mechanics in the anesthetized rat. J Appl Physiol 1978;45:255-260.

21 Knelson JH, Howatt WF, DeMuth GR: Effect of respiratory pattern on alveolar gas exchange. J Appl Physiol 1970;29:328-331.

22 Mercat A, Diehl JL, Michard F, Anguel N, Teboul JL, Labrousse J, Richard C: Extending inspiratory time in acute respiratory distress syndrome. Crit Care Med 2001;29:4044
23 Aström E, Uttman L, Niklason L, Aboab J, Brochard L, Jonson B: Pattern of inspiratory gas delivery affects $\mathrm{CO}_{2}$ elimination in health and after acute lung injury. Intensive Care Med 2008;34:377-384

24 Fernandez R, Mendez M, Younes M: Effect of ventilator flow rate on respiratory timing in normal humans. Am J Respir Crit Care Med 1999;159:710-719.

$\checkmark 25$ Kondili E, Prinianakis G, Anastasaki M: Acute effects of ventilator settings on respiratory motor output in patients with acute lung injury. Intensive Care Med 2001;27: 1147-1157.

26 Dimitriou G, Greenough A, Laubscher B, Yamaguchi N: Comparison of airway pressure-triggered and airflow-triggered ventilation in very immature infants. Acta Paediatr 1998;87:1256-1260.

27 Hayashi F, Yoshida A, Fukuda Y, Honda Y: $\mathrm{CO}_{2}$-ventilatory response of the anesthetized rat by rebreathing technique. Pflugers Arch 1982;393:77-82.

28 Saetta M, Mortola JP: Breathing pattern and $\mathrm{CO}_{2}$ response in newborns rats before and during anesthesia. J Appl Physiol 1985;58: 1988-1986.

29 Stunden CE, Filosa JA, Garcia AJ, Dean JB, Putnam RW: Development of in vivo ventilatory and single chemosensitive neuron responses to hypercapnia in rats. Respir Physiol 2001;127:135-155.

>30 Viale JP, Duperret S, Mahul P, Delafosse B, Delpuech C, Weismann D, Annat G: Time course evolution of ventilatory responses to inspiratory unloading in patients. Am J Respir Crit Care Med 1998;157:428-434.

31 Corne S, Webster K, Younes M: Hypoxic respiratory response during acute stable hypocapnia. Am J Respir Crit Care Med 2003;167: 1193-1199.

32 Sinderby C, Beck J, Spahija de Marchie M, Lacroix J, Navalesi P, Slutsky AS: Inspiratory muscle unloading by neurally adjusted ventilatory assist during maximal inspiratory efforts in healthy subjects. Chest 2007;131: 711-717. 